\title{
Positive rolandic sharp waves in the EEG of the premature newborn: a five year prospective study
}

\author{
Stéphane Marret, Dominique Parain, Emmanuel Jeannot, Danièle Eurin, Claude Fessard
}

\begin{abstract}
In a prospective study of 301 premature newborn infants, neonatal tracings were done to evaluate the use of the electroencephalogram (EEG) and positive rolandic sharp waves (PRSW) in the diagnosis and prognosis of periventricular leucomalacia. Each infant had ultrasonographic studies and standardised neurological examinations at 1 year of age or later. Two hundred and eighty infants were followed up at 1 year.

This study demonstrated that the absence of PRSW was correlated with a favourable motor development (98.2\%) and confirmed the great value of PRSW in the diagnosis and the prognosis of periventricular leucomalacia. PRSW were sensitive (98\%) and specific (84\%) markers of developmental motor disability and were a sensitive $(96 \%)$ marker of severe spastic diplegia. A frequency above $2 /$ minute was a specific $(92 \%)$ sign of severe spastic diplegia. Social and language developmental abnormalities were not correlated with the neonatal EEG.
\end{abstract}

Periventricular leucomalacia and intraventricular haemorrhage are serious neurological complications of prematurity. Numerous studies of infants prospectively scanned with ultrasound have shown that intraventricular haemorrhage had a relatively benign prognosis in the absence of involvement of the brain parenchyma or ventricular dilation ${ }^{12}$ whereas periventricular leucomalacia had a worse one. ${ }^{1-3}$ Though intraventricular haemorrhage is readily recognised by transfontanellar ultrasonography, early positive diagnosis and prognosis of periventricular leucomalacia are not always so easy.

Positive rolandic sharp waves (PRSW) on the electroencephalogram (EEG) were first reported by Cukier et al in 1972 in premature newborn infants with intraventricular haemorrhage. ${ }^{4}$ $\mathrm{We}^{5}$ and others ${ }^{6-8}$ observed that PRSW were not always associated with intraventricular haemorrhage but rather with white matter necrosis or periventricular leucomalacia. PRSW seemed to be, therefore, a marker of periventricular leucomalacia rather than of intraventricular haemorrhage. From these anecdotal reports we decided to conduct a formal prospective evaluation of the use of the EEG and detection of PRSW in the diagnosis and prognosis of periventricular leucomalacia in premature newborn infants.

\section{Subjects and methods}

\section{SUBJECTS}

The study was carried out over a five year period between 1 January 1986 and 31 December 1990 on all newborn infants of gestational age less than 33 weeks who were born between 1 January 1986 and 31 December 1989 and admitted to the department of neonatalogy or the intensive care unit of the Centre Hospitalier Universitaire de Rouen; 350 newborns were included. Forty nine died before 3 months and were then excluded from the study. Three hundred and one survived and had at least one EEG within the first 14 days of life; most had two or three EEGs before the age of 1 month.

\section{ELECTROENCEPHALOGRAPHY}

Two hundred and fifty five infants had three EEGs. The first EEG was done between days 2 and 4, the second between days 7 and 14, and the third between days 21 and 30 . Twenty eight infants had two EEGs and 18 only one. The recordings were done at the infant's bedside and lasted at least 45 minutes. They were performed with a portable 10 or 16 channel machine (Alvar) (six or 10 derivations for EEG, one for an electrocardiogram, one for ocular movements, one for an electromyogram, and one or three for respiratory movements). Bipolar montage was adapted for the newborn infant. The scalp electrodes were put in T4-02, FP2C4, C4-02, FP1-C3, C3-01, T3-01 in the 10 channel machine montage and in FP2-T4, T4-02, FP2-C4, C4-02, FP1-C3, C3-01, FP1-T3, T3-01 in the 16 channel machine montage. The EEGs were analysed by two of the authors (SM, DP) and another board certified paediatric neurologist. As previously described the PRSW (fig 1) were identified as sharp transients of positive polarity appearing in the rolandic regions (C3-4). ${ }^{6-9}$ For each record-

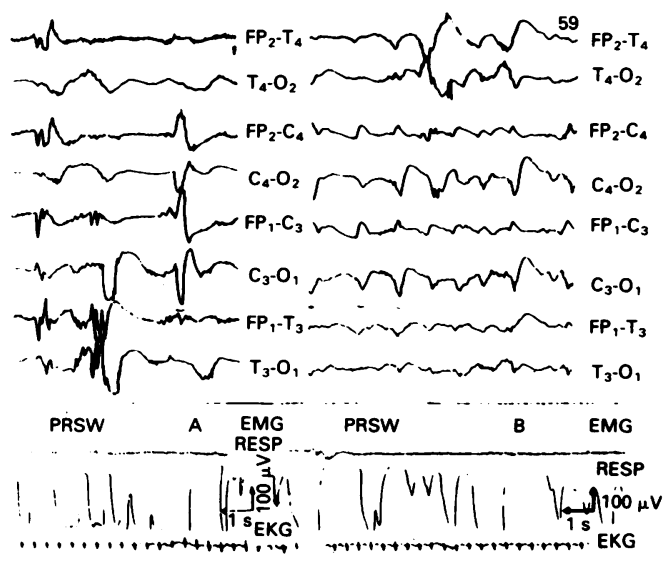

Figure 1 Example of PRSW type $A$ and type $B$ in an EEG made on a 16 channel machine. 
ing, PRSW were classified as type A (isolated and sharply differentiated from the background activity) or B (occurring in bundles and less well differentiated from background activity) according to Blume and Dreyfus-Brisac. ${ }^{10}$ The frequency (number of PRSW per minute of recording time) was calculated over all the tracing. When it was possible, the persistence of PRSW in two EEGs was noted. Background activity was classified as normal or mildly, moderately, or markedly abnormal, according to the Clancy and Tharp criteria. ${ }^{9}$

\section{ULTRASONOGRAPHIC STUDIES}

Each infant had two transfontanellar ultrasonographic studies: one in in the first and another in the third week. They were performed with a real time linear array ultrasound scanner (Sonel 400 ) with a 5 or $7.5 \mathrm{MHz}$ tranducer. Coronal and sagittal views of brain were obtained. They were seen by a paediatric radiologist without knowledge of the clinical and EEG data. Intraventricular haemorrhage, intraparenchymal haemorrhage, and periventricular cysts in the white matter were studied. Intraventricular haemorrhage was classified as type II when there was no ventricular dilation and type III when there was dilation. Intraparenchymal haemorrhage was considered when there was a unilateral marked echodensity with coexisting intraventricular haemorrhage. Germinal layer haemorrhages were not taken into account and neither were increased parenchymal hypoxicischaemic echodensities as infants had only two ultrasonographic studies at a two week interval. In our study, only periventricular cysts were considered as a certain sign of periventricular leucomalacia.

\section{FOLLOW UP}

Standardised neurological and developmental examinations were carried out at $3,6,9$, and 12 months at least and at 24 months in the older infants. They were classified into three motor developmental groups: I: always normal; II: transient or minor motor developmental abnormalities with hand free sitting acquired at 1 year and walking independently acquired at 2 years; and III: severe motor abnormalities with spastic diplegia or quadriplegia. Social and language development was evaluated by Brunet-Lezine tests at 1 and 2 years. ${ }^{11}$ It was abnormal below 75 , normal above. Because of the duration of follow up standardised measurement of cognitive development was not obtained.

\section{STATISTICS}

Different groups were compared by the $\chi^{2}$ test, with Yates's correction or Fisher's exact test, as appropriate; $p$ values were considered significant below 0.05 . When there was a significant difference in comparing groups for one criteria, sensitivity, specificity, predictive positive and negative value, and constant $k$ of relative risk were calculated.

\section{Results}

ELECTROENCEPHALOGRAPHIC ANALYSIS

Eight hundred and thirty nine EEGs were analysed. PRSW were observed in 114 of the 301 infants (38\%). The first PRSW were seen within the first 14 days in 106 infants $(93 \%)$. Eighty $(70 \%)$ had both types A and B in the same recording or in different recordings. Six had only type A and 28 type B. Frequency is shown in fig 2. Persistence of PRSW was noted in two recordings at least in $63 / 113(56 \%)$ of cases with two or three EEGs and PRSW.

Thirty five (12\%) infants had an abnormal background (eight mild, 19 moderate, eight marked). Twenty two of the 35 had an abnormal background and PRSW (eight mild, nine moderate, five marked). In 33 infants an abnormal background was seen in the first EEG. In only two of these 33 did the background abnormality persist in the second recording. Two infants had a moderately abnormal activity only in the second EEG.

\section{ULTRASONOGRAPHIC STUDIES}

Twenty one infants (7\%) had an intraventricular haemorrhage, eight of the 21 with a dilation (type III). There was no intraparenchymal haemorrhage. Eighteen $(6 \%)$ had periventricular cysts. Three of the 18 with cysts also had an intraventricular haemorrhage.

\section{OUTCOME}

At 1 year of age 21/301 infants were lost to follow up. Two hundred of the 280 had a normal motor development (group I). Eighty were neurologically impaired at follow up: 52 had transient or minor motor abnormalities (group II) and 28 a severe spastic diplegia or quadriplegia (group III).

Prevalence of impaired motor development in infants followed up within the first year was $28 \cdot 6 \%$. Prevalence of severe diplegia or quadriplegia (group III) was $10 \%$. Developmental quotient for social and language development was low in 20 infants. Three infants had impaired development without spastic diplegia, and 17 had associated diplegia or quadriplegia.

Two hundred and fifteen infants of the 280 were investigated at 2 years. Forty infants were lost to follow up. Forty six did not reach 2 years of age. One hundred and thirty six of the 215 were normal. Of the remainder three had

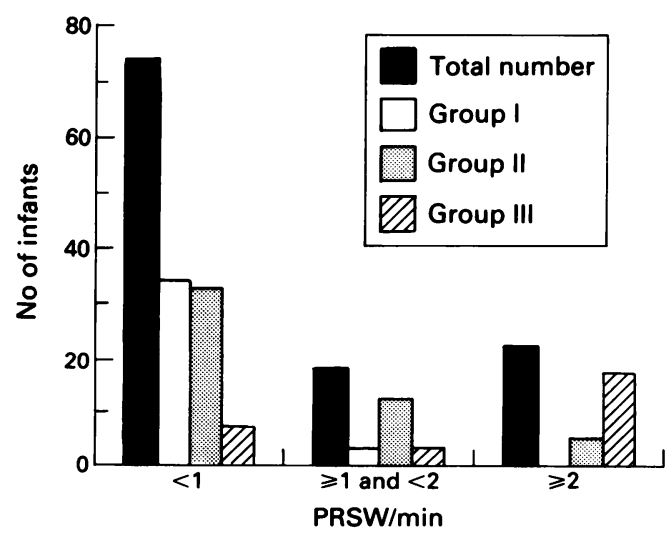

Figure 2 Electroencephalography: the first column shows the total number of infants in each PRSW frequency group and the last three columns the distribution of PRSW frequency in each motor development group. 
impaired development without motor impairment, 48 belonged to group II, and 28 to group III.

EEG AND ULTRASOUND

PRSW were present in all 18 children with cystic periventricular leucomalacia shown by ultrasound. Thirteen of the $21(62 \%)$ infants with intraventricular haemorrhage (10 type II and three type III) had PRSW. Eighty six of the 114 infants with PRSW had normal ultrasound scans.

EEG AND OUTCOME

The distribution of PRSW in the groups followed up is shown in fig 3 . Altogether $98.2 \%$ of the infants without PRSW had normal motor development. Sixty eight per cent of the infants with PRSW and only $1.8 \%$ of the infants without had impaired motor development. The absence of PRSW was a sensitive and specific sign of right motor development (sensitivity= $81 \%$, specificity $=96 \%$ ). The PRSW were very sensitive $(98 \%)$ and specific $(84 \%)$ markers of impaired motor outcome (group II plus III) and were sensitive markers of severe motor handicap (96\%) (group III) but less specific (65\%). An infant with PRSW had 33 times the risk of having a severe spastic diplegia than an infant without PRSW. Thirty seven infants with PRSW had no delay in motor development. Three infants had no PRSW but an abnormal motor development (two a moderate one, one a severe one). The infant with no PRSW and severe diplegia had only two EEGs in neonatal period (one at D2 and one at D24).

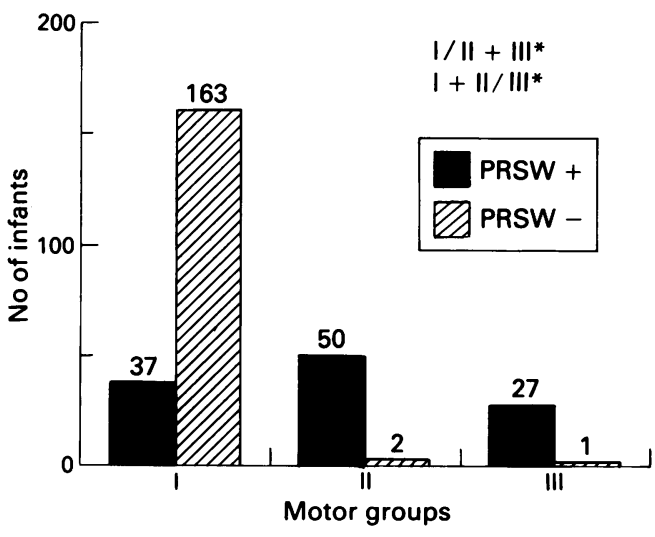

Figure 3 Electroencephalography in follow up groups showing number of infants with or without PRSW in each motor development group; " $p<0 \cdot 00001$.
Frequency above 2/minute was statistically different between group III compared with groups I plus II ( $p<0.00001$ ) (fig 2). It had a good specificity as a bad prognosis marker (97\%) but a low sensitivity (50\%). Among the 37 infants with PRSW but normal motor development, PRSW appeared less than 2 /minute in all and less than $1 /$ minute in 34 cases. Neither persistence of PRSW nor type A or B were significant prognostic markers.

There was no correlation between abnormal background and language and social development in our study.

EEG, ULTRASOUND, AND OUTCOME

The results are shown in the table. In the infants with impaired motor development (group II and III), PRSW on the neonatal EEG were more frequent than cysts seen on ultrasound. The infants with an intraventricular haemorrhage and PRSW (62\%) were in the three motor developmental groups whereas the infants with an intraventricular haemorrhage and without PRSW (38\%) were all in the group I.

\section{Discussion}

A lot of studies have shown the importance of sequential ultrasound scans in the diagnosis of periventricular leucomalacia. ${ }^{1-3}{ }^{12}$ Our prospective study confirms the great value of an EEG associated with ultrasound in the diagnosis and the prognosis of periventricular leucomalacia.

In our study the incidence of PRSW (38\%) is comparable with that of Lacey et al $(37 \%) .{ }^{13}$ The absence of PRSW on the neonatal EEG is correlated with unimpaired motor development $(98.2 \%)$ whereas in their presence impaired development is more frequent $(68 \%)$. PRSW are a good, sensitive, and specific diagnostic marker of impaired motor outcome. They are a good, sensitive diagnostic marker of severe motor abnormalities (group III) but not a specific one. Such motor abnormalities are known to be due to periventricular leucomalacia. ${ }^{14}$ Periventricular leucomalacia can be seen on ultrasound when there is development of cysts. But it is noteworthy that hypoxicischaemic parenchymal lesions can be undiagnosed by ultrasound when there is a diffuse gliosis or small areas of periventricular leucomalacia. ${ }^{15}$ In our study, 18 of the 80 infants with impaired motor outcome had cysts. Therefore in premature newborns PRSW are a useful sign of neonatal periventricular ischaemia with impaired motor development.

Outcome, PRSW, and lesions seen on ultrasound

\begin{tabular}{|c|c|c|c|c|c|c|}
\hline \multirow{2}{*}{$\begin{array}{l}\text { Ultrasound } \\
\text { appearances }\end{array}$} & \multicolumn{2}{|l|}{ Group I } & \multicolumn{2}{|l|}{ Group II } & \multicolumn{2}{|c|}{ Group III } \\
\hline & PRSW- & PRSW+ & PRSW- & PRSW+ & $P R S W-$ & PRSW+ \\
\hline \multicolumn{7}{|l|}{ Cysts: } \\
\hline Without & 163 & 37 & 2 & 47 & 1 & 12 \\
\hline With & 0 & 0 & 0 & 3 & 0 & 15 \\
\hline \multicolumn{7}{|c|}{$\begin{array}{l}\text { Intraventricular } \\
\text { haemorrhage: }\end{array}$} \\
\hline Without & 155 & 32 & 2 & 46 & 1 & 23 \\
\hline $\begin{array}{l}\text { With } \\
\text { (type III) }\end{array}$ & $\begin{array}{c}8 \\
(5)\end{array}$ & 5 & 0 & $\begin{array}{c}4 \\
(1)\end{array}$ & 0 & $\begin{array}{l}4^{*} \\
(2)\end{array}$ \\
\hline
\end{tabular}

*3/4 also had cysts. 
Some infants have PRSW but no motor sequelae. None of these infants have a high frequency of PRSW ( $\geqslant 2 /$ minute); this is the single most specific marker of severe impaired motor development in our study. These infants will also probably have some neonatal white matter ischaemic lesions. Perhaps they will later have cognitive developmental abnormalities, minimal brain dysfunction, and problems with educational achievement.

PRSW were first reported by Cukier $e t$ al in premature infants with intraventricular haemorrhage. ${ }^{4}$ As reported by Lacey $e t$ al in their study of infants with intraventricular haemorrhage, some had PRSW and others did not. ${ }^{13}$ The infants with PRSW and intraventricular haemorrhage (with or without dilation) have a worse motor development than those without PRSW, who are always normal. In the former case intraventricular haemorrhage is associated with ischaemic parenchymal lesions, which are a more critical determinant of outcome than the amount of haemorrhage in the ventricle. ${ }^{7}$ Three of these infants also had evidence of cysts seen on ultrasound.

Unlike other reported findings, ${ }^{7} 1316$ background abnormalities were not a prognostic sign in our study. Marked and persistent background abnormalities are usually bad prognostic markers. ${ }^{13} 16$ Several facts can explain the lack of significance in our study. Firstly, it concerned only infants who survived the neonatal period and secondly, the infants were all less than 33 weeks of gestational age. In these immature babies, the cerebral cortex is spared the effects of ischaemia because of its rich leptomeningeal arterial supply. ${ }^{17}$ In our study one infant later developed infantile epilepsy and one West's syndrome. Finally, the eight children with marked background EEG abnormalities all had excessive interburst activity lasting for more than a minute. They never persist in two EEGs. Only one infant with marked background abnormalities is impaired for social and language development. Only two infants have persistent, but moderate, background abnormalities; both are normal.

This study demonstrates the need for serial EEGs in the neonatal period in premature newborn infants. They are useful for predicting both favourable and unfavourable outcome.

We thank Dr D Samson-Dollfus for valuable advice and analysis of the EEG, Dr $\mathrm{N}$ Moore for valuable editorial comments Dr J Menard, for statistical analysis of the data, and C Berland for skilful technical assistance.

1 De Vries LS, Dubowitz LMS, Dubowitz V, et al. Predictive value of cranial ultrasound in the newborn baby: a reappraisal. Lancet 1985; ii: 137-40.

2 Stewart AL, Reynolds EOR, Hope PL, et al. Probability of neurodevelopmental disorders estimated from ultrasound appearance of brain of very preterm infants. Dev Med Child Neurol 1987;29:3-11

3 Fawer CL, Diebold P, Calame A. Periventricular leucomalacia and neurodevelopmental outcome in preterm infants Arch Dis Child 1987;62:30-6.

4 Cukier F, Andre M, Monod N, Dreyfus-Brisac C. Apport de l'EEG au diagnostic des hemorragies intraventriculaire du prématuré. Revue d'Electroencephalographie et de Neurophysiologie Clinique 1972;2:318-22.

5 Marret S, Parain D, Samson-Dollfus D, et al. Positive rolandic sharp waves and periventricular leukomalacia in the newborn. Neuropediatrics 1986;17:199-202.

6 Lombroso CT. Neonatal electroencephalography. In: Niedermayer, E, Lopes da Silva F, eds. Electroencephalography. Baltimore: Urban and Schwazenberg, 1982: graphy.

7 Bejar R, Coen RW, Meritt TA, et al. Focal necrosis of the white matter (periventricular leucomalacia): sonographic, pathologic, and electroencephalographic features. $A f N R$ 986;7:1073-80.

8 Novotny EJ, Tharp BR, Coen RW, et al. Positive rolandic sharp waves in the EEG of the premature infant. Neurology 1987;37:1481-6.

9 Clancy RP, Tharp B. Positive rolandic sharp waves in the electroencephalogram of premature neonates with intraventricular hemorrhage. Electroencephalogr Clin Neurophysiol 1984;57:395-404.

10 Blume WT, Dreyfus-Brisac C. Positive rolandic sharp waves in the neonatal EEG. Electroencephalogr Clin Neurophysiol 1982;53:277-82.

11 Brunet $\mathrm{O}$, Lezine I. Le développment psychologique de la première enfance. 2nd Ed. Paris: Presse Universitaire de France, 1966.

12 Dubowitz LMS, Bydder GM, Mushin V. Developmental sequence of periventricular leukomalacia. Arch Dis Child sequence of perive

13 Lacey DJ, Topper WH, Buckwald S, et al. Preterm very-low birth weight neonates: relationship of EEG to intracrania hemorrhage, perinatal complications and developmental outcome. Neurologv 1986;36:1084-7.

14 Banker B, Larroche JC. Periventricular leukomalacia of infancy. Arch Neurol 1962;7:386-410

15 Hope P, Gould SJ, Howards S, et al. Precision of ultrasound diagnosis of pathologically verified lesions in the brains of very preterm infants. Dev Med Child Neurol 1988;30: very pr-71.

16 Tharp BR, Scher MS, Clancy RR. Serial EEGs in normal and abnormal infants with birth weights less than 1200 grams. A prospective study with long term follow up. NeuropediaA prospective study
trics $1989 ; 20: 64-71$.

17 De Reuck J, Chattma AS, Richardson EP. Pathogenesis and evolution of leukomalacia in infancy. Arch Neurol 1972;27: 229-36. 